\title{
Do early paternal exposures to lifestyle factors such as smoking increase the risk of chronic diseases in the offspring?
}

\author{
Adelheid Soubry, Geert Verbeke and Cathrine Hoyo
}

European Journal of Human Genetics (2014) 22, 1341-1342; doi:10.1038/ ejhg.2014.206; published online 8 October 2014

$\mathrm{T}$ he results of a recent epidemiological study on the effects of paternal smoking on children's body composition published in $E J H G^{1}$ contribute to the limited research in humans on environmentally induced risk for chronic diseases in the next generation(s) through the father. Inherited non-genetic changes may be an evolutionary response to adapt relatively quickly to environmental variations. However, if an individual is exposed to an adverse environmental exposure, such as a new pollutant, chemical, or pathogen, during a critical developmental stage in life, the risk of developing an endocrine, metabolic, or mental disorder or even cancer in the offspring may increase. Thus far, most research in this area focuses on maternal contributions, while a potential transgenerational epigenetic effect through the father has generally been underexplored. The Avon Longitudinal Study of Parents and Children (ALSPAC), led by Pembrey, showed that adolescent sons of fathers who started smoking before puberty are at high risk of being obese. ${ }^{1}$ Although no underlying biological mechanism has been shown yet, this fascinating finding suggests that cigarette smoke metabolites may induce epigenetic changes during prepubertal production of

Professor A Soubry is at the Epidemiology Research Group, $\mathrm{KU}$ Leuven, Leuven, Belgium; Professor G Verbeke is at I-Biostat, KU Leuven, Leuven, Belgium and Professor C Hoyo is at the Department of Biological Sciences, NC State University, Raleigh, NC, USA and at the Department of Obstetrics and Gynecology, Duke University Medical Center, Durham, NC, USA

E-mail: adelheid.soubry@hotmail.com spermatogonia in the testes. Remarkably, in order to persist until the offspring's early adulthood, this epigenetic information needs to be carried on through all developmental processes, including spermatogenesis, conception, and embryonic growth. An earlier historical Swedish study by the same authors showed a similar transgenerational response to food availability. The longevity of men was been exposed to an excess of food during the slow growth period, before the prepubertal growth peak. ${ }^{2}$ These gender- and timespecific associations suggest that the epigenome of the male germ line is malleable by environmental factors at specific timepoints in life. An epigenetic link in humans was shown for the first time in the Newborn Epigenetic Study (NEST); this NC-based birth cohort showed that imprint-regulatory regions of newborns are differentially methylated in response to paternal obesity. 3,4

The theory on environmentally induced epigenetic transgenerational inheritance of disease susceptibility through the male germ line has been supported through a number of animal experiments on male exposures to various factors such as environmental toxins (insecticides, bisphenol-A, jet-fuel, etc.) and dietary components (alcohol, folate, genistein, high-fat or low-protein diet). Susceptibility time windows for such environmentally induced epigenetic changes through the paternal germ line have recently been reviewed. ${ }^{5}$ Although animal models provide a useful tool to explore the individual effects of each environmental component through decreased if their paternal grandfathers had maternal or paternal exposures at different timepoints, human responses to these particular components may be different. Extrapolating the findings from rodents to humans is often difficult. Dose-response may be different and the human natural environment contains more components that may interact with the one under study. Moreover, temporal differences in expression patterns of genes during early development as well as in maturation of germ cells complicate translation of animal data to humans. Hence, epidemiological studies are essential, but difficult to perform, especially if multiple generations are needed to explore.

The study by Northstone et al ${ }^{1}$ suggests that paternal cigarette smoking causes a stable 'signature' in the germ line, especially if the exposure occurred before puberty. However, further investigations are needed to confirm these findings, via other data sets, but also through refined analyses of the ALSPAC data. ALSPAC includes repeated measurements of the same children at different ages, from 7 to 17 years. Northstone et al performed crosssectional analyses at each age separately, and did not explore the information present in the longitudinal trends. This is worrisome since considerable loss of follow-up is observed, apparently related to BMI, but potentially also to gender or unmeasured factors, which often leads to bias. ${ }^{6}$ The data of Northstone et al highlight that paternal smoking before the age of 11 years is associated with increased BMI, waist circumference, and total body fat mass of adolescent sons. Raw BMI scores alone in children are generally difficult to interpret. However, findings on fat mass and waist circumference were significant and encouraging, but they were mainly based on less than 20 sons aged 13 and 15 years, and no data were collected on waist circumference at age 17. Furthermore, a systematic comparison between different ages of paternal smoking is lacking; the reference group included an equal number of fathers who started smoking after the age of 11 years and non-smoking fathers, and the specificity test on adjusted data was only performed in the category of fathers who started smoking between 11 and 13 years of age (Supplementary Table 8). Here too, sample sizes were limited, and no results were shown for children's BMI. The study further presumes a potential trend in smoking behavior in young children from fathers who smoked at young age (Supplementary Table 6). Although $P$-values did not reach statistical significance, presumably because of the small samples, it is recommended to include personal smoking by children as a 
potential confounder. Northstone et al adjusted for 'child-smoking' in one of their analyses (Supplementary Table 7) and showed that this did not change the overall conclusion for boys. However, their data indicated that if smoking in daughters was included in their regression analyses the earlier detected association between paternal smoking at age $<11$ and waist circumference or fat mass at age 13 was attenuated (compared to Table 3 ). This remarkable finding corresponds with the finding of Pasch et $a l^{7}$ in a longitudinal study, showing that smoking at a young age predicts an increase in body fat percentage a few years later; however, sex differences were not examined. The differences shown between the sons and daughters in the data of Northstone et al remain unexplained, but sample sizes were often small. Moreover, no formal interaction tests for differences between boys and girls were discussed.
We conclude that although the ALSPAC study indicates that timing of onset of paternal smoking, especially at pre-puberty, is important for the next generation's health, the information present in this study has not been fully explored. Further research is needed to confirm the harmful health-related effects of paternal lifestyle on the next generation. Current epidemiological studies only show the tip of the iceberg, but the field of epigenetic inheritance is evolving. It is promising for a better understanding of the effects of several common exposures at certain stages in paternal life and their risks of developing diseases in the offspring.

\section{CONFLICT OF INTEREST}

The authors declare no conflict of interest.
1 Northstone K, Golding J, Davey Smith G, Miller LL, Pembrey M: Prepubertal start of father's smoking and increased body fat in his sons: further characterisation of paternal transgenerational responses. Eur J Hum Genet 2014; 22: 1382-1386.

2 Pembrey ME, Bygren LO, Kaati G et al: Sex-specific, male-line transgenerational responses in humans. Eur $J$ Hum Genet 2006; 14: 159-166.

3 Soubry A, Schildkraut JM, Murtha A et al: Paternal obesity is associated with IGF2 hypomethylation in newborns: results from a Newborn Epigenetics Study (NEST) cohort. BMC Med 2013; 11: 29.

4 Soubry A, Murphy SK, Wang F et al: Newborns of obese parents have altered DNA methylation patterns at imprinted genes. Int J Obes (Lond) 2014; e-pub ahead of print 26 November 2013; doi:10.1038/ijo.2013.193.

5 Soubry A, Hoyo C, Jirtle RL, Murphy SK: A paternal environmental legacy: evidence for epigenetic inheritance through the male germ line. Bioessays 2014; 36 : 356-371.

6 Verbeke G, Molenberghs GLinear Mixed Models for Longitudinal Data, Springer Series in Statistics Springer-Verlag: New York, 2000.

7 Pasch KE, Velazquez CE, Cance JD, Moe SG, Lytle LA: Youth substance use and body composition: Does risk in one area predict risk in the other? J Youth Adolescence 2012; 41: 14-26. 